\title{
Floating Hemispherical Helical Antenna: Analysis of Gain, Efficiency and Resonant Frequency
}

\author{
Zia M. LONI, Hugo G. ESPINOSA, David V. THIEL \\ School of Engineering and Built Environment, Griffith University, Brisbane, Australia \\ h.espinosa@griffith.edu.au
}

Submitted July 8, 2018 / Accepted August 27, 2018

\begin{abstract}
This paper reports the effect of seawater conductivity on gain, efficiency and resonant frequency of a hemispherical helical antenna. The size of the copper ground plane for the hemispherical antenna can be reduced using conductive seawater as part of the ground plane for the antenna. Seawater increases the gain from $6 \mathrm{dBi}$ to $8 \mathrm{dBi}$ but with a decreased efficiency. The simulated radiation efficiency of the antenna on water surface is $61 \%$. This paper also reports the design of a low cost floating buoy. The buoy provides a waterproof setup for the circuitry and antenna. The buoy can be effectively used for shallow water coastal monitoring.
\end{abstract}

\section{Keywords}

Hemispherical antenna, vacuum forming, floating buoy, ocean sensors, 3D printing, Specific Absorption Rate (SAR), Wireless Sensor Networks (WSN)

\section{Introduction}

Recent technological advancement in oceanic engineering has helped in exploring the ocean world. The focus is not only to explore the sea bed but to protect the oceans from increased human activity resulting in water pollution [1], [2]. An effective way to perform ocean monitoring is through sensor networks [3]. Ocean sensors can communicate using acoustic, light or electromagnetic (EM) radiation. High frequency (HF) EM waves can be used to design small size and low cost sensor nodes which can effectively monitor shallow water coastal regions. Seawater parameters including salinity, conductivity, permittivity and temperature are interdependent and are governed by permittivity models [4]. Ocean communication using EM waves in the HF range is not possible with completely submerged transceivers due to the high conductivity of seawater resulting in high attenuation [5], [6]. Shammaa et al. conducted experiments at $\mathrm{MHz}$ frequencies and concluded that through-water propagation is only possible with very high transmission power [5]. Floating sensor nodes can achieve significant propagation range with low transmit power exploiting surface wave propagation which suffers less attenuation compared to through-water propagation [7].
Loni et al. achieved a propagation range of $35 \mathrm{~m}$ using an insulated wire floating monopole antenna working at $433 \mathrm{MHz}$ with transmit power of $10 \mathrm{dBm}$ [7], [8]. Jackson et al. performed experiments at $2.45 \mathrm{GHz}$ with floating sensors and achieved a propagation range of $25 \mathrm{~m}$ with transmit power of $4 \mathrm{dBm}$ [9]. Seawater in the near field of the antenna affects its resonant frequency, gain and efficiency. The shift in resonant frequency is due to the high permittivity of seawater. James et al. performed experiments at different frequency ranges to record the shift in resonant frequency [10]. He reported that the shift in resonance is more significant at lower frequency and becomes less significant as the frequency is increased. The type of antenna used with sensor nodes needs to be effective since the ocean environment is constantly changing and power levels are fluctuating. A hemispherical antenna has small size and compact structure and so is a good substitute for the monopole antenna. Hemispherical antennas are designed on conducting ground plane which is large compared to the radius of the hemisphere [11], [12]. Hemispherical antennas have also been addressed by combining slot and monopole or even a single monopole optimizing the vertical and horizontal component so as to radiate as a half-sphere [13], [14]. The size of the ground plane is not suitable for electrically small sensor nodes. This paper reports how seawater conductivity can be used to our advantage if the sea surface forms part of ground plane of a hemispherical antenna. The result is a reduced size of the ground plane and consequently small sized sensor nodes. The change in antenna characteristics from the seawater is dependent on the magnitude of antenna exposure to seawater environments as well as the sea surface roughness.

Figure 1 shows the design of a floating buoy holding both the antenna and the circuit board electronics. Vacuum forming and 3D printing technologies were used for the construction of the buoy. These technologies give the advantages of low cost manufacturing and design freedom. Vacuum forming is used to build structures using a thermoplastic sheet. In this case, the sheet thickness was $2 \mathrm{~mm}$ and the material was Polyethylene Terephthalate Glycol (PETG). Under elevated temperature and reduced pressure, the plastic takes the shape of a mould and becomes a firm structure upon cooling. Permittivity and loss tangent of PETG at $1 \mathrm{MHz}$ are reported as 2.4 and 0.2 , respectively. 


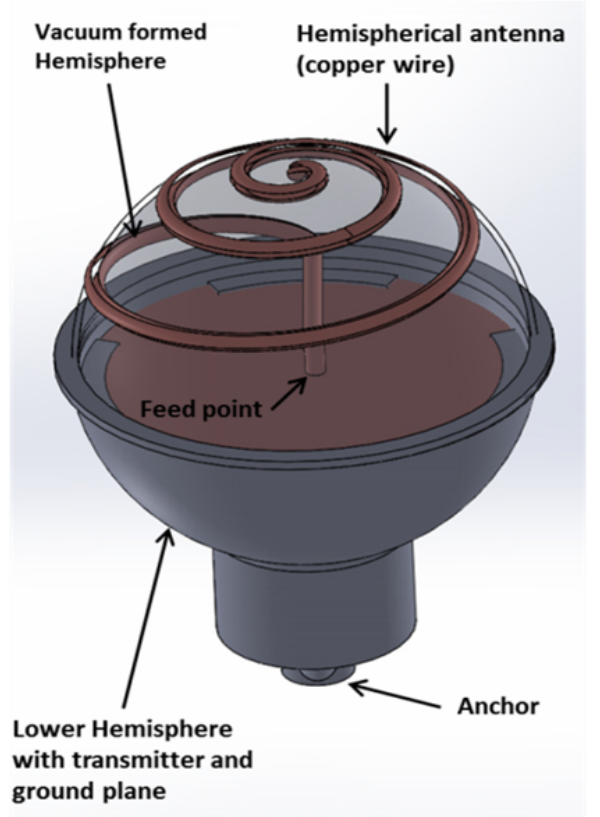

Fig. 1. A spherical floating sensor buoy contains a copper ground plane on the horizontal axis. The upper transparent hemisphere contains the three and half turn Hemispherical antenna on 3D printed ABS material. The lower hemisphere contains the sensor electronics.

PETG is stronger and more reliable as compared to other available $3 \mathrm{D}$ printing materials. In addition, it can be printed in transparent structures. Due to lower manufacturing time, any complex structure can be built and modified more rapidly [15]. Recently, 3D printing has also become popular to build low cost structures. The material commonly used for printing are Acrylonitrile butadiene styrene (ABS) and Polylactic Acid (PLA). Vacuum formed and $3 \mathrm{D}$ printing have the added advantage of providing water proof setup which is a requirement for seawater applications. A floating buoy must be waterproof and needs protection from severe environment hazards including rain and tides. The designed sensor buoy (Fig. 1) is air filled. A circular copper ground plane lies horizontally on the axis of the sphere where the upper hemisphere is the antenna and the lower hemisphere contains the electronics and sensing equipment. The complete system is waterproof and can be used for monitoring water quality in shallow water coastal areas [7]. The principal direction of the radiation pattern of the hemispherical antenna is directed in the vertical direction which is advantageous for collecting data through satellite or Unmanned Air Vehicles (UAV's).

The paper is organized as follows: Section 2 discusses hemispherical antenna design and construction of the floating buoy. Section 3 describes the simulations and measurement results of antenna on water surface. Section 4 discusses the conclusion and future work.

\section{Antenna and Buoy Design}

Figure 1 shows a three and a half turn hemispherical antenna mounted on a floating buoy. The antenna design was optimized in CST software [16]. The resonant frequency was controlled by adjusting the radius of the sphere. The parametric equation for the hemispherical antenna is given in [11], [17], where $\theta$ is the angle from the vertical axis and $\phi$ is the angle in the horizontal plane. The total length of the antenna $L$ is given by

$$
\begin{gathered}
r=a, \\
\theta=\cos ^{-1}\left(\frac{\phi}{N \pi}\right), 0 \leq \phi \leq N \pi, \\
L=\int_{0}^{\pi} a \sqrt{1+(N \pi)^{2} \sin ^{4} \theta} \mathrm{d} \theta
\end{gathered}
$$

where $N$ represents the number of turns and $a$ is the radius of the sphere. The diameter of the ground plane is $120 \mathrm{~mm}$. Equation (3) gives the length of the helical wire forming the hemispherical antenna which is equal to $79.2 \mathrm{~cm}$. The radiating element of the antenna is a single conductor insulated copper wire. The antenna is fed from the center by extending a wire from the side to the center of the hemisphere. The length of the center feed is equal to the radius of the hemisphere.

Figure 2 shows a vacuum formed structure for holding the antenna. In vacuum forming, as the heated plastic takes the shape of the mould under test, a 3D printed hemisphere with antenna indentations was designed in SolidWorks [18], using the same design parameters as that used in CST modeling. The mould was placed in the vacuum forming apparatus under differential pressure and elevated temperature. Due to the tracks on the printed hemisphere, the same tracks appeared on the inner surface of the plastic hemisphere in the form of narrow indentations. The radiating element (copper wire, see Fig. 1 and Fig. 2) was glued into this indentation. This structure protects the antenna from seawater and weather conditions.

The lower part of the hemisphere holding the low power transmitter and ground plane was 3D printed with ABS material. Figure 1 and Figure 3 show the lower hemisphere which contains the ground plane and a low power

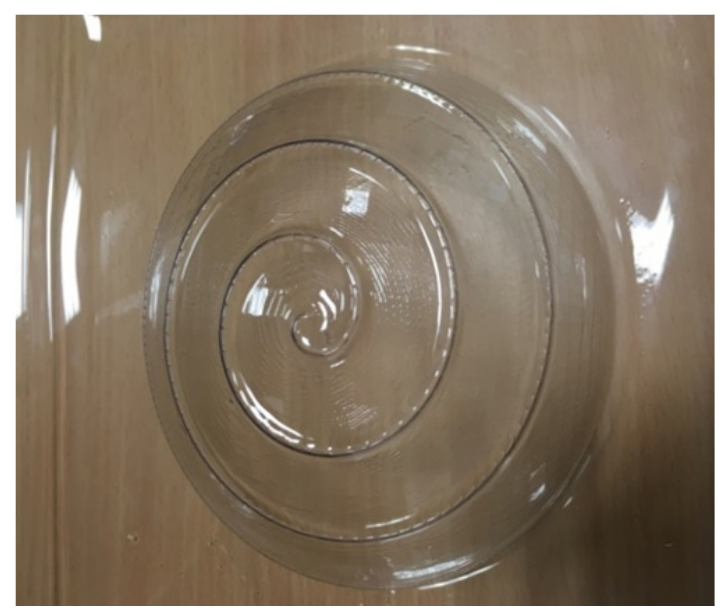

Fig. 2. Vacuumed formed hemisphere with small width canals on the inner surface. 


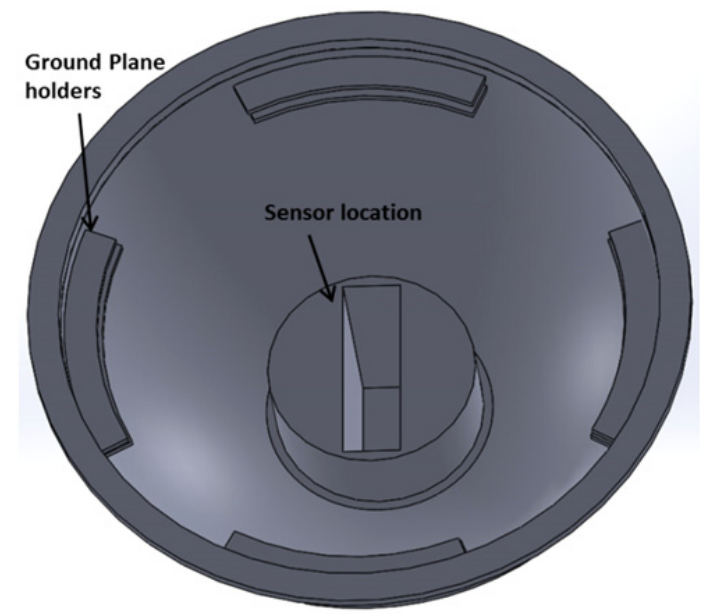

Fig. 3. Inner structure of the lower hemisphere with location for sensors and the antenna ground plane.

transmitter. To improve the stability and to maintain orientation of the floating sensor buoy, an anchor location was also printed to add suitable weights. The upper and lower hemispheres were combined using a gasket which was also $3 \mathrm{D}$ printed. Given the upper hemisphere is optically transparent, photovoltaic cells can be placed on the ground plane for additional power of the sensor.

\section{Measurements}

\subsection{Laboratory Measurements}

Figure 4 shows the setup for return loss measurements carried over a water surface in the laboratory. The measurements were performed in a plastic container with saline water. Sodium chloride $(\mathrm{NaCl})$ was added until a conductivity of $5 \mathrm{~S} / \mathrm{m}$ was achieved. The copper wire (antenna) was glued to the indentation on the inner surface of the upper hemisphere. The antenna coaxial feed was fixed to the bottom of the container and the cable was connected to a Portable Vector Network Analyzer (N9923A Field Fox Handheld RFVNA@6GHz) through a SMA connector.

Two experiments were performed using a slightly modified arrangement between them. In the first experiment, the saline solution was used as a ground plane. This was achieved using an earth wire with one end connected to the SMA connector and the other end was exposed to the saline water (see Fig. 5). In the second experiment, the hemispherical antenna was placed on a small copper ground plane floating in the saline water. The coaxial cable and SMA connector were pulled through a sealed pipe to protect the feed point from direct contact with the saline solution. The copper ground plane was insulated from the water.

The measurements were performed over a frequency range of 0 to $1 \mathrm{GHz}$. The saline solution was gradually poured in the water tub and the change in the return loss was measured. The measurements were recorded until the saline solution reached the lowest turn of the hemisphere.

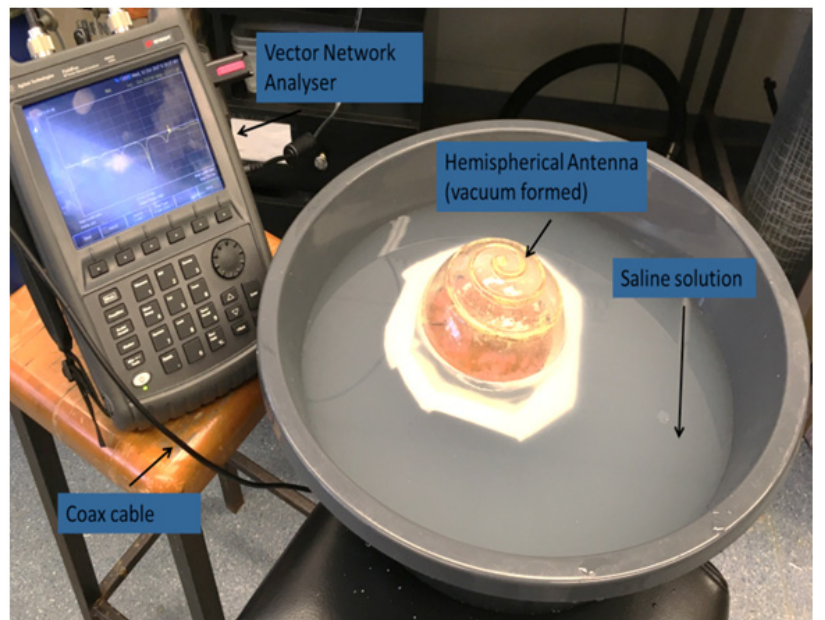

Fig. 4. Setup for return loss measurements. The coaxial feed cable is fed through the bottom of the plastic container. The water conductivity was $5 \mathrm{~S} / \mathrm{m}$.

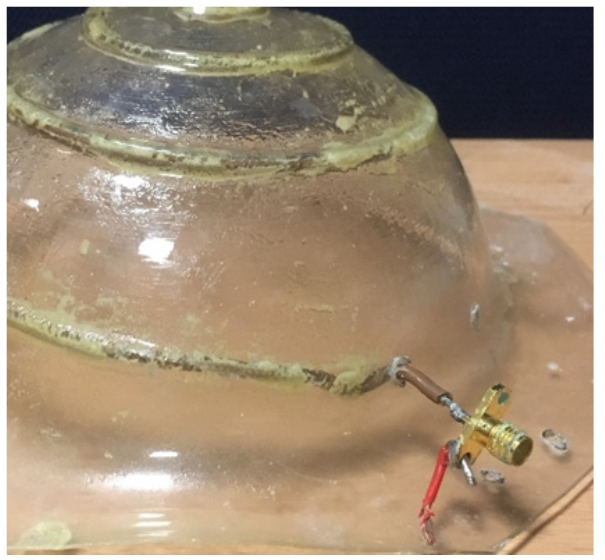

Fig. 5. Saline solution used as ground plane. One end of the earth wire was attached to SMA connector and the other end was exposed to saline water.

The conductivity level was monitored with a WP-81 conductivity meter from TPS Pty Ltd.

\subsection{Results}

Figure 6 shows the simulated and measured results when saline water was used as a ground plane for the antenna. Simulations showed a return loss of $-30 \mathrm{~dB}$ at $465 \mathrm{MHz}$. The measured resonant frequency was recorded as $429 \mathrm{MHz}$ with a return loss of $-38 \mathrm{~dB}$. The simulated radiation efficiency for the antenna on the water surface was calculated as $19.5 \%$. These results show that water as ground plane results in a significant reduction of radiation efficiency. This is too low for most practical applications. To increase the antenna efficiency, the copper ground plane with diameter $120 \mathrm{~mm}$ was included under the hemispherical antenna. The antenna with copper ground plane was first tested in air and then floating on the water surface.

Figure 7 shows the comparison of simulated and measured results for the antenna with copper ground plane. The simulated and measured resonant frequencies in air were recorded as $772 \mathrm{MHz}$ and $765 \mathrm{MHz}$, respectively. On 


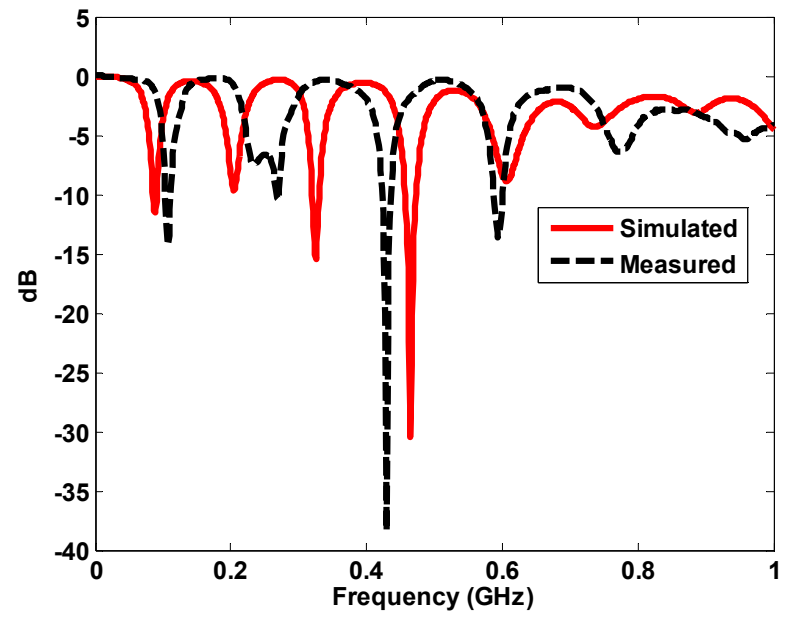

Fig. 6. Simulated and measured return loss measurements with seawater as ground plane.

the water surface, the simulated and measured resonances were $723 \mathrm{MHz}$ and $740 \mathrm{MHz}$, respectively. The frequency shift in the measured frequencies was $25 \mathrm{MHz}$, however; for simulated results it was calculated as $50 \mathrm{MHz}$. We can also observe that the water surface increased the bandwidth of the antenna. The simulated radiation efficiency of the antenna over the water surface was calculated as $61 \%$. A comparison between the two experimental results (Fig. 6 and Fig. 7) shows that the antenna on the water surface is matched to a lower frequency, i.e. $429 \mathrm{MHz}$. The introduction of the copper ground plane shifted the resonance to a higher frequency. The shift in resonance is due to the different surface impedance given by the seawater and the copper ground plane. The complex permittivity $\left(\varepsilon_{\mathrm{r}}\right)$ of the saline solution is a function of the operating frequency [4]. The permittivity values at $429 \mathrm{MHz}$ and $740 \mathrm{MHz}$ were calculated as $71-206 \mathrm{j}$ and $71-121 \mathrm{j}$, respectively [4], [7].

Figure 8 shows the simulated radiation pattern for the antenna arrangements discussed above. Ideally, a hemispherical antenna on an infinite ground plane would radiate in the vertical direction $\left(\theta=0^{\circ}\right)$ with no back lobe $\left(\theta=180^{\circ}\right)$. Simulation results for the antenna with copper ground plane in air shows a large back lobe. The reason is the finite size of the copper ground plane. The same antenna when placed on the water surface results in reduction of the back lobe with more radiation directed in the vertical direction [19]. The saline solution behaves as a lossy ground plane, although there is no physical contact between the saline solution and the copper ground plane. The lowest gain was calculated when the saline solution was used as the ground plane. The reason for the low gain is the high radiation absorption by seawater which reduces the radiation efficiency to less than $20 \%$. These results show that the saline solution alone cannot be used as a ground plane. However, a combination of the copper ground plane and the seawater increases the gain at the cost of decreasing efficiency compared with an infinitely large conducting sheet. The size of the copper ground can be increased to improve the radiation efficiency. The copper ground $(120 \mathrm{~mm})$ increased the efficiency to $61 \%$ making it more suitable for installation in a sensor node. The size of the

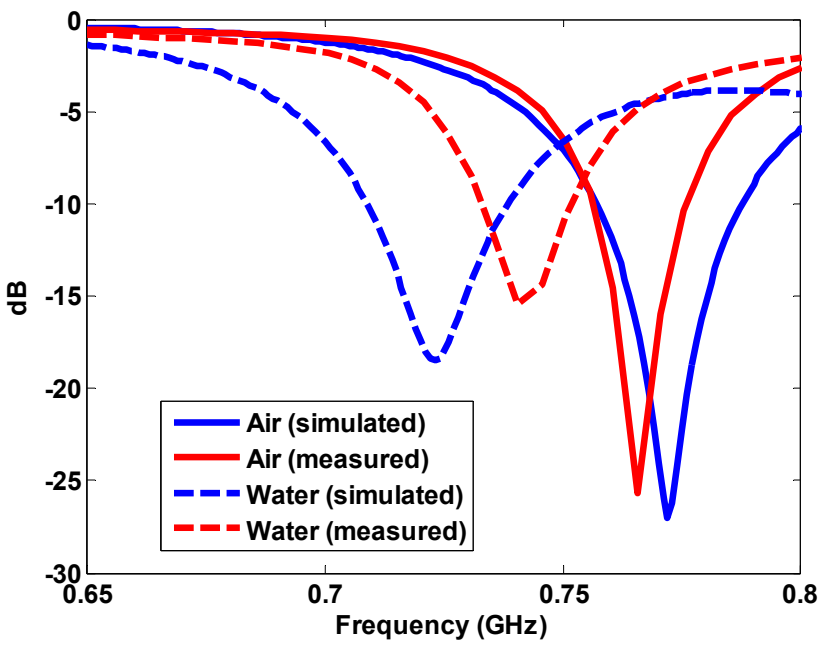

Fig. 7. Simulated and measured reflection coefficient $\left(S_{11}\right)$ for the hemispherical antenna in air and with the ground plane $(120 \mathrm{~mm})$

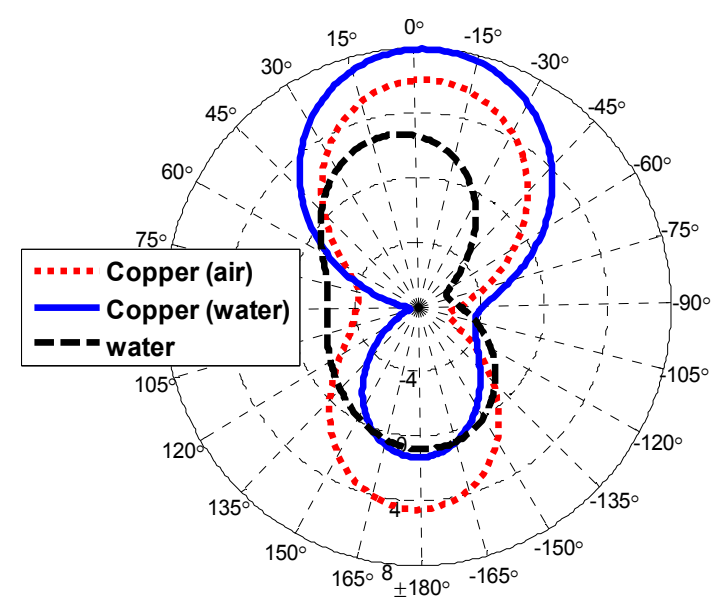

Fig. 8. Simulated radiation pattern of the sensor buoy in air and over the saline water surface.

ground plane and radiation efficiency can be traded off depending on the application of the sensor node. Increasing the size would increase the efficiency of the antenna but the size of sensor node would increase too. This is not desirable for our ocean monitoring applications.

This antenna can achieve a significant propagation range with a low power transmitter. Figure 9 shows the prototype design for the floating buoy mounted with the hemispherical antenna. A low powered transmitter and copper ground plane are located within the sensor buoy. The design is waterproof, however; it can easily be opened to replace batteries and other electronic equipment. The buoy can be anchored by adjusting the weights located at the bottom of the structure.

The radiation pattern of the hemispherical antenna (see Fig. 8) is directed in a vertical direction with an $8 \mathrm{~dB}$ decrease in the back lobe radiation and $2 \mathrm{~dB}$ increase in the vertical direction. This is advantageous for data collection from the floating sensor nodes. Real time information from the sensors can be retrieved by an Unmanned Air Vehicle (UAV) flying over the sensor nodes or through remote sensing utilizing communication satellites. 


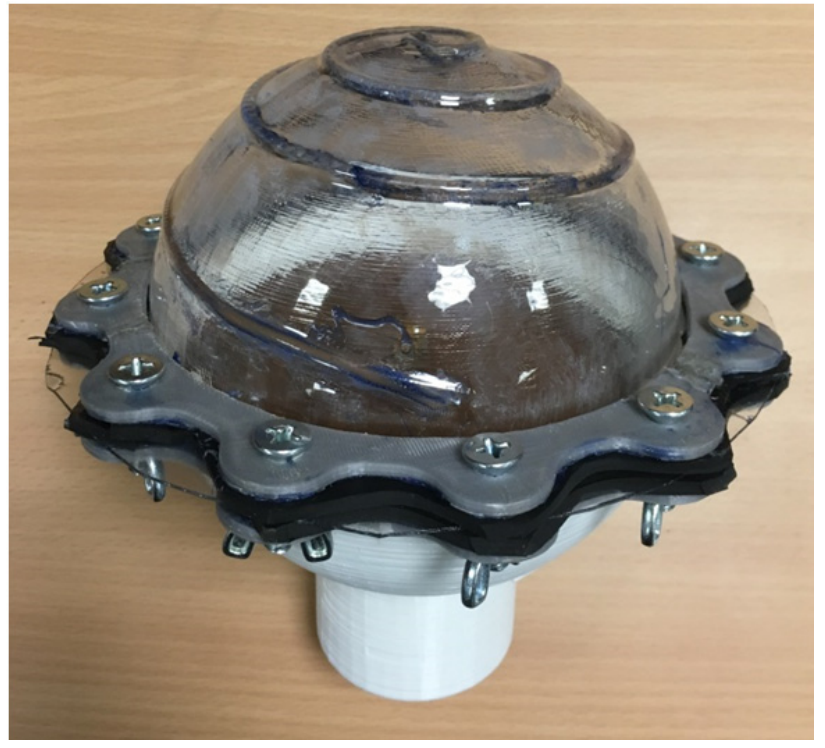

Fig. 10. Prototype of vacuum formed hemisphere with antenna glued to the small width canals on the inner surface.

\section{Conclusion}

Vacuum forming and 3D printing techniques were used to manufacture a low cost floating buoy prototype. The vacuum formed hemisphere protects the hemispherical helical antenna from environmental hazards which includes rain, high tides and ocean waves. The buoy includes a hemispherical antenna with a small ground plane. Seawater was partially used as a ground plane which increased the gain of antenna. The highly conductive seawater has resulted in transforming the radiation pattern which gives us the liberty to use a small sized ground plane. The simulated radiation efficiency was $61 \%$. The designed buoy is low cost and can easily be modified to accommodate sensors for collecting information from oceanic environment. The propagation range measurements can be performed with low power transmitter $(10 \mathrm{dBm})$ which will influence the density (nodes per $\mathrm{m}^{2}$ ) of the sensor network. The radiation pattern of the antenna is directed in the vertical direction which is advantageous for communication with satellites or passing UAV's.

\section{References}

[1] LIU, L., ZHOU, S., CUI, J. Prospects and problems of wireless communication for underwater sensor network. Wireless Communication and Mobile Computing, special issue on Underwater Sensor Networks: Architectures and Protocols, 2008, no. 8, p. 977-994. DOI: 10.1002/wcm.654

[2] WEBSTER, T., LEMKERT, C. J. Sediment resuspension within a micro tidal estuary/embayment and the implication to channel management. Journal of Coastal Research, 2002, vol. 36, Special Issue International Coastal Symposium (ICS 2002), p. 753-759. DOI: $10.2112 / 1551-5036-36 . s p 1.753$

[3] HEIDEMANN, J., STOJANOVIC, M., ZORZI, M. Underwater sensor networks: applications, advances and challenges,
Philosophical Transactions of the Royal. Society A, 2012, vol. 370, p. 158-175. DOI: $10.1098 /$ rsta.2011.0214

[4] KLEIN, L., SWIFT, C. T. An improved model for the dielectric constant of seawater at microwave frequencies. IEEE Transactions on Antennas and Propagation, 1977, vol. 25, no. 1, p. 104-111. DOI: $10.1109 /$ JOE.1977.1145319

[5] AL SHAMMAA, A. I., SHAW, A., SAMAM, S. Propagation of electromagnetic waves at $\mathrm{MHz}$ frequencies through seawater. IEEE Transactions on Antennas and Propagation, 2004, vol. 52, no. 11, p. 2843-2849. DOI: 10.1109/TAP.2004.834449

[6] ABDOU, A., SHAW, A., MASON, A., et al. Wireless sensor network for underwater communication. In IET Conference on Wireless Sensor Systems. London (UK), 2012, p. 3-8. DOI: 10.1049/cp.2012.0579

[7] LONI, Z. M., ESPINOSA, H. G., THIEL, D. V. Floating monopole antenna on a tethered subsurface sensor at $433 \mathrm{MHz}$ for ocean monitoring applications. IEEE Journal on Oceanic Engineering, Oct 2017, vol. 42, no. 4, p. 818-825. DOI: 10.1109/JOE.2016.2639111

[8] LONI, Z. M., ESPINOSA, H. G., THIEL, D. V. Insulated wire fed floating monopole antenna for coastal monitoring. Radioengineering, April 2018, vol. 27, no. 1, p. 127-133. DOI: 10.13164/re.2018.0127

[9] JACKSON, N. C., THIEL, D. V. ISM band $2.45 \mathrm{GHz}$ propagation studies in a coastal environment. In International Antenna Propagation Symposium Tasmania. Australia, 2015, p. 663-666. ISBN: 978-4-8855-2302-1

[10] JAMES, D. A., GALEHAR, A., THIEL, D. V. Mobile sensor communications in aquatic environments for sporting applications Procedia Engineering, 2010, vol. 2, no. 2, p. 3017-3022. DOI: 10.1016/j.proeng.2010.04.104

[11] SAFAAI-JAAZ, A., CARDOSO, J. C. Radiation characteristics of a spherical helical antenna. IEE Proceedings Microwaves, Antennas and Propagation, 1996, vol. 143, no. 1, p. 7-12. DOI: 10.1049/ip-map: 19960037

[12] HUI, T. H., CHAN, Y. K., YUNG, N. K. E. The input impedance and the antenna gain of the spherical helical antenna. IEEE Transactions on Antennas and Propagation, 2001, vol. 49, no. 8, p. 1235-1237. DOI: $10.1109 / 8.943319$

[13] LONG, S. A. A combination of linear and slot antennas for quasiisotropic coverage. IEEE Transactions on Antennas and Propagation, 1975, vol. 23, no. 4, p. 572-576. DOI: 10.1109/TAP.1975.1141121

[14] ANDUjar, A., ANGUERA, J., PUENTE, C., PEREZ, A. On the radiation pattern of L-shaped wire antennas. Progress in Electromagnetic Research M, 2009, vol. 6, p. 91-105. DOI: 10.2528/PIERM09012204

[15] NKOMO, N., GWAMURI, J., SIBANDA, N. R., NKIWANE, L. A study of applications of 3D printing technology and potential applications in the plastic thermoforming industry. IOSR Journal of Engineering, 2017. ISSN (e): 2250-3021, ISSN (p): 2278-8719.

[16] CST MICROWAVE STUDIO, Comput. Simulation Tech., Munich, Germany: Simulia (TM) Dassault Systemes, 2016.

[17] LONI, Z. M., ESPINOSA, H. G., THIEL, D. V. Floating hemispherical helical antenna for ocean sensor networks. IEEE Journal on Oceanic Engineering, 2018, p. 1-8. DOI: 10.1109/JOE.2018.2853198

[18] SOLIDWORKS, Massachusetts, USA, Dassault SystemesSolidWorks Corporation, 2017.

[19] LONI, Z. M., ESPINOSA, H. G., THIEL, D. V. Vertically directed microwave radiation from a floating hemispherical antenna. In Australian Microwave Symposium (AMS). Brisbane (Australia), 2018, p. 45-46. DOI: 10.1109/AUSMS.2018.8346974 


\section{About the Authors ...}

Zia M. LONI received his bachelor degree in Telecommunications Engineering from the University of Engineering and Technology Peshawar, Pakistan, in 2006, the master's degree from Mohammad Ali Jinnah University Islamabad, Pakistan, in 2010, in Electronics Engineering. He is currently working towards his $\mathrm{PhD}$ degree at Griffith University, Brisbane, Australia. He is also working as a sessional instructor in the School of Engineering, Griffith University. From 2010 to 2015, he has been a lecturer with COMSATS Institute of Information Technology, Abbottabad, Pakistan. His research interests include electromagnetic wave propagation, antennas, remote sensing, and wireless sensor networks.

Hugo G. ESPINOSA received the degree in Electronics and Telecommunications Engineering from the Monterrey Institute of Technology and Higher Education, Mexico, in 1998, the master's degree from the University of Sao Paulo, Brazil, in 2002, and the Ph.D. degree (summa cum laude) from the Technical University of Catalonia, Spain, in 2008, both in Electrical Engineering. He joined the Federal Polytechnic School of Lausanne, Switzerland, in 2006, for a four-month research stay. From 2009 to 2010, he has been a Post-Doctoral Fellow with the Physical Electronics Department, School of Electrical Engineering, Tel Aviv
University, Israel. Since 2011, he has been with the School of Engineering at Griffith University, Brisbane, Queensland, Australia, where he is currently a Lecturer in Electronic Engineering. His research interests include computational electromagnetics, wave propagation, electromagnetic geophysics, wireless sensor networks, and wearable sensor technology for human monitoring. Dr. Espinosa serves as a Senior Member of the IEEE.

David V. THIEL received the degree in Physics and Applied Mathematics from the University of Adelaide, Adelaide, SA, Australia, and the master's and Ph.D. degrees from James Cook University, Townsville, QLD. He is currently the Deputy Head of School (Research) with Griffith University, Brisbane, QLD, Australia. He is a Fellow of the Institution of Engineers, Australia, and a Chartered Professional Engineer in Australia. His research interests include electromagnetic geophysics, sensor development, electronics systems design and manufacture, antenna development for wireless sensor networks, environmental sustainability in electronics manufacturing, sports engineering, and mining engineering. He authored the book Research Methods for Engineers, and co-authored a book on Switched Parasitic Antennas for Cellular Communications. Prof. Thiel has authored six book chapters, over 130 journal papers, and has co-authored more than nine patent applications. 